OPEN ACCESS

Edited by:

Kathiravan Purmal,

Columbia Asia Hospital, Malaysia

Reviewed by:

Ola B. Al-Batayneh,

Jordan University of Science and

Technology, Jordan

Arlette Setiawan,

Universitas Padjadjaran, Indonesia

*Correspondence:

Hanyao Huang

huanghanyao_cn@scu.edu.cn

Caixia Gong

gongcaixia01@163.com

tThese authors have contributed equally to this work

Specialty section

This article was submitted to Pediatric Surgery,

a section of the journal

Frontiers in Pediatrics

Received: 23 December 2021

Accepted: 20 January 2022

Published: 14 February 2022

Citation:

Xie Z, Yang C, Zhao Y, Yang Y, Xia W,

Zong $Y$, Chi T, Shi B, Huang $H$ and

Gong C (2022) Anxiety in Chinese Patients With Cleft Lip and/or Palate:

A Preliminary Study

Front. Pediatr. 10:842470.

doi: 10.3389/fped.2022.842470

\section{Anxiety in Chinese Patients With Cleft Lip and/or Palate: A Preliminary Study}

\author{
Zhuojun Xie ${ }^{\dagger}$, Chao Yang ${ }^{\dagger}$, Yuxi Zhao, Yichun Yang, Weiyao Xia, Yuan Zong, Ting Chi, \\ Bing Shi, Hanyao Huang* and Caixia Gong*
}

State Key Laboratory of Oral Diseases and National Clinical Research Center for Oral Diseases and Department of Oral Maxillofacial Surgery, West China Hospital of Stomatology, Sichuan University, Chengdu, China

Objectives: To preliminarily analyze factors that affected the prevalence of anxiety in Chinese patients with cleft lip and/or palate (CL/P).

Methods: The Generalized Anxiety Disorder Scale (GAD-7) was used to screen anxiety in Chinese CL/P patients. Non-CL/P individuals were also included as the control group. Sociodemographic and clinical data consisting of diagnosis, gender, only child or not, monthly household income, and current family location were collected to analyze possible factors that could affect the anxiety of this patient population.

Results: One hundred forty-two and 78 valid questionnaires were collected from the study and control groups, respectively. The mean GAD-7 score of the study group (3.092 \pm 3.381$)$ was significantly lower than the control (3.987 \pm 2.505$)$. Moreover, the proportion of patients presenting with moderate-severe anxiety was larger in the study group than in the control group (6.6 vs. 0.0\%). Statistically significant differences in GAD-7 scores were observed between the study and control groups when the patient was the only child, living in an urban area, or the monthly household income was between 1,000 and 5,000 yuan.

Conclusion: Although the severity of anxiety in Chinese CL/P patients was not severer than those without CL/P, there was a relatively high incidence of moderate-severe anxiety in CL/P patients, while the only child, current family location and the monthly household income played significant roles in affecting anxiety psychology.

Keywords: cleft lip and/or palate, anxiety, GAD-7, psychology, Chinese CL/P patients

\section{INTRODUCTION}

Cleft lip and palate (CL/P) is one of the most common congenital craniofacial abnormalities, characterized by failure of normal fusion of the palate and lip at the midline during embryonic development, resulting in a clinically obvious deformity of the newborn (1), bringing unignorable appearance malformations and increasing risk for various psychological diseases like loneliness, low self-esteem, social disorders, and mood disorders to patients (2). Although there lies a strong association between psychological wellbeing and appearance, and between psychological wellbeing and social adjustment (3), some other studies demonstrated few differences in psychological status between CL/P patients and those who without appearance malformations (4). It reminds us of the need for further studies. 
As a common mental illness, anxiety refers to a feeling of nervousness, impatience, and restlessness about things, accompanied by physical symptoms such as palpitation, chest distress, and hyperventilation (5). It has been reported that compared with individuals without $\mathrm{CL} / \mathrm{P}, \mathrm{CL} / \mathrm{P}$ patients are easily faced with relatively higher severity of anxiety (6). As anxiety could affect the life quality of the patients, identifying factors influencing anxiety status in Chinese CL/P patients would provide the theoretical basis for psychological intervention and improve the quality of cleft care. Thus, in this study, we sought to preliminarily investigate the characteristics of anxiety in Chinese $\mathrm{CL} / \mathrm{P}$ patients by applying the Generalized Anxiety Disorder Scale (GAD-7) to analyze the effect of CL/P on anxiety and the possible related influencing factors.

\section{MATERIALS AND METHODS}

\section{Subjects}

Chinese Patients with a history of cleft lip and (or) cleft palate who visited West China Hospital of Stomatology, Sichuan University, between July 2019 and January 2021 were enrolled in the study group. Inclusion criteria of the study group were as follows:

- Patients with complete or incomplete unilateral or bilateral non-syndromic cleft lip and (or) palate.

- Patients who underwent cleft lip repair and (or) palatoplasty.

- Patients aged 10 years or above (7).

Through the distribution of questionnaires in the general population, inclusion criteria of the control group were as follows:

(1). Individuals with no significant facial defects or other major diseases.

(2). Individuals aged 10 years or above (7).

Sociodemographic and clinical data were collected for analysis, including diagnosis, gender, only child or not, monthly household income, and current family location.

The research protocol was reviewed and approved by the Ethics Committee of West China Hospital of Stomatology, Sichuan University (No. WCHSIRB-D-2016-084R1). Written informed consents were acquired from all the patients or their parents.

\section{Measurement of Anxiety Status}

The Generalized Anxiety Disorder 7-Item Scale (GAD-7) was used to assess patient anxiety status. Seven items were used to assess the frequency of anxiety symptoms over 2 weeks on a 4-point Likert scale ranging from 0 (never) to 3 (nearly every day). The total score of GAD-7 ranged from 0 to 21 , with increasing scores indicating more severe functional impairments due to anxiety. The total score was calculated for each patient and interpreted as follows: normal (0-4), mild (5-9), moderate (10-14), and severe (15-21).

As one of the most widely used assessment scales applied for screening, diagnosis, and severity assessment of anxiety disorders (8), GAD-7 has been transadapted into Chinese culture and validated (9). It can serve as an effective tool for anxiety screening in Chinese adolescents (7).

In present study, all subjects were given questionnaires in Chinese version and guided by trained volunteers to make sure every item was fully understood. Each subject completed the questionnaire independently.

\section{Statistical Analysis}

SPSS 23.0 (IBM Corp., Armonk, NY, USA) is used for statistical analyses, and the count data was represented by mean \pm standard deviation (SD). Independent samples-T test was used to compare the two independent variables within groups and the independent variables between groups, while one-way ANOVA was used to compare more-than-two independent variables within groups when the compliance with normal distribution is tested by Shapiro-Wilk. The Kruskal-Wallis test was typically used when the normality assumption was violated. $P$-values $<$ 0.05 were statistically significant, while $P$-values $<0.01$ were highly statistically significant.

\section{RESULTS}

\section{Participant Characteristics}

A total of 145 GAD-7 (142 valid and 3 invalid) questionnaires were collected from the study group (Mean age: $17.2 \pm 5.8$ years), while a total of 78 questionnaires were collected from the control group (Mean age: $18.5 \pm 5.2$ years). No significant difference in age was found between the two groups. The demographic characteristics of both groups were shown in Table $\mathbf{1}$.

TABLE 1 | The demographic characteristics of the study group and control group.

\begin{tabular}{lcccc}
\hline Variables & \multicolumn{2}{c}{ The study group } & \multicolumn{2}{c}{ The control group } \\
\cline { 2 - 5 } & Number & Percentage & Number & Percentag \\
\hline 1. Diagnosis & & & & \\
Cleft lip and palate & 81 & $57.0 \%$ & & \\
Cleft lip & 38 & $26.8 \%$ & & \\
Cleft palate & 23 & $16.2 \%$ & & \\
2. Gender & & & & \\
Male & 87 & $61.3 \%$ & & \\
Female & 55 & $38.7 \%$ & & \\
3. Only child & & & & \\
Only child & 45 & $31.7 \%$ & 45 & $57.7 \%$ \\
Non-only child & 97 & $68.3 \%$ & 33 & $42.3 \%$ \\
4. The monthly household income & & & \\
Under 1,000 yuan & 27 & $19.0 \%$ & 2 & $2.6 \%$ \\
1,000-5,000 yuan & 73 & $51.4 \%$ & 27 & $34.6 \%$ \\
5,000-10,000 yuan & 17 & $12.0 \%$ & 28 & $35.9 \%$ \\
More than 10,000 yuan & 18 & $12.7 \%$ & 21 & $26.9 \%$ \\
5. Current family location & & & & \\
Rural & 73 & $51.4 \%$ & 17 & $21.8 \%$ \\
Urban & 69 & $48.6 \%$ & 61 & $78.2 \%$ \\
& & & &
\end{tabular}




\section{Anxiety Status in the Study and Control Groups}

The mean GAD-7 scores in the study and control groups were 3.092 and 3.987 points, respectively, and the scores difference was statistically significant (Table 2 ). Moreover, the proportion of moderate-severe anxiety in the study group was larger than in the control group (6.6 vs. $0.0 \%$ ) (Table 3 ).

\section{The Analyses of the Factors That Influenced GAD-7 Scores}

Sociodemographic and clinical data consisting of diagnosis, gender, only child or not, monthly household income, and current family location did not influence the anxiety status between subgroups in both study and control groups. However, analysis between groups showed a statistically significant difference in subjects that were an only child and lived in an urban location, while a highly statistically significant difference was demonstrated in subjects with a total family income between 1,000 and 5,000 yuan (Table 4).

\section{DISCUSSION}

It has always been assumed that facial appearance is vital for healthy psychosocial development (10). Given that CL/P patients experience difficulties in feeding, speech dysfunction, and cosmetic defects from birth, their psychological development could be affected (11). However, in contrast, other studies demonstrated no significant difference in psychological status between CL/P patients and non-CL/P individuals $(12,13)$. In the setting of the fact that research on the incidence of anxiety and its influencing factors of CL/P patients in China were considerably unclear, the present research aimed to gain a deeper insight into the prevalence of anxiety in Chinese CL/P patients and identify potential influencing factors.

Interestingly, we found that the average GAD-7 score of Chinese CL/P patients was lower than that of the control group.
Possible explanations were as follows: There were studies that claimed CL/P patients reported better emotional wellbeing and overall self-worth than healthy people, and the impact of CL/P was not worse than having other concerns around appearance $(14,15)$, given that CL/P reportedly strengthened the individual's vulnerability to disadvantageous psychological experiences when faced with emotional difficulties and negative self-perception, as most of the patients were observed to perform and adapt well when confronted with adversities $(16,17)$. What's more, as the severity of anxiety could be divided into normal, mild anxiety, moderate anxiety and severe anxiety on the basis of GAD-7 score, we found that the proportion of moderate-severe anxiety in the study group was larger than that in the control group, indicating that CL/P disease might pose a severer negative effect on patient's anxiety. Nonetheless, further studies with larger sample sizes should be conducted to substantiate the potential negative effect exerted by CL/P.

The heterogeneity of cleft types could explain the differences observed in our study. Although the difference was not statistically significant, higher GAD-7 scores were found in patients diagnosed with cleft palate (CP) or cleft lip and palate (CLP) than those with cleft lip (CL), consistent with previous studies. As for CLP patients, they faced more significant challenges in social interactions and communications than other types of CL/P resulting from significant speech difficulties and language delays (18), potentially contributing to the worse psychological status. Additionally, it had been investigated that children with $\mathrm{CP}$ showed severer problems with parent- and teacher-reported depression, anxiety, and other psychological difficulties than children with CL or CLP, partially explained by greater speech problems and subsequently poorer learning disorder rather than facial defects because of its morphological abnormities and functional defects.

We also found that female patients scored slightly higher in GAD-7 than males while the difference was not statistically significant, indicating the possibility that female patients experienced relatively worse psychological conditions to some

TABLE 2 | The GAD-7 scores of the study group and control group.

\begin{tabular}{lcccccc}
\hline & Mean \pm SD & Median & Range & Interquartile Range & \multicolumn{2}{c}{ 95\% Confidence interval for mean } \\
\cline { 5 - 6 } & & & & & Lower bound & Upper bound \\
\hline The study group & $3.092 \pm 3.381$ & 2.000 & 17.000 & 3.000 & 2.531 & 3.653 \\
The control group & $3.987 \pm 2.505$ & 4.000 & 9.000 & 4.000 & 3.423 & 4.552
\end{tabular}

${ }^{*} P<0.05$ by independent samples- $T$ test.

TABLE 3 | Severity of anxiety in the study and control groups.

\begin{tabular}{|c|c|c|c|c|c|c|c|c|c|}
\hline & \multirow[t]{2}{*}{ Total } & \multicolumn{2}{|c|}{ Normal } & \multicolumn{2}{|c|}{ Mild anxiety } & \multicolumn{2}{|c|}{ Moderate anxiety } & \multicolumn{2}{|c|}{ Severe anxiety } \\
\hline & & Number & Percentage & Number & Percentage & Number & Percentage & Number & Percentage \\
\hline The study group & 142 & 110 & $77.5 \%$ & 24 & $16.9 \%$ & 6 & $4.2 \%$ & 2 & $1.4 \%$ \\
\hline The control group & 78 & 46 & $59.0 \%$ & 32 & $41.0 \%$ & 0 & $0.0 \%$ & 0 & $0.0 \%$ \\
\hline
\end{tabular}


TABLE 4 | Factors affecting anxiety status of Chinese patients with CL/P.

\begin{tabular}{|c|c|c|c|c|c|}
\hline \multirow[t]{2}{*}{ Variables } & \multicolumn{2}{|c|}{ The study group } & \multicolumn{2}{|c|}{ The control group } & \multirow[t]{2}{*}{$P$-value (study vs. control) } \\
\hline & Mean \pm SD & $P$-value & Mean \pm SD & $P$-value & \\
\hline \multicolumn{6}{|l|}{ 1.Diagnosis } \\
\hline Cleft lip and palate & $3.123 \pm 3.455$ & 0.967 & & & \\
\hline Cleft lip & $2.974 \pm 3.234$ & & & & \\
\hline Cleft palate & $3.174 \pm 3.499$ & & & & \\
\hline \multicolumn{6}{|l|}{ 2.Gender } \\
\hline Male & $3.046 \pm 3.316$ & 0.841 & & & \\
\hline Female & $3.164 \pm 3.511$ & & & & \\
\hline \multicolumn{6}{|l|}{ 3.Only child } \\
\hline Non-only child & $3.412 \pm 3.665$ & 0.081 & $3.636 \pm 2.421$ & 0.292 & 0.729 \\
\hline Only child & $2.326 \pm 2.597$ & & $4.244 \pm 2.560$ & & $0.017^{\star}$ \\
\hline \multicolumn{6}{|c|}{ 4.The monthly household income } \\
\hline Under 1,000 yuan & $4.037 \pm 4.808$ & 0.064 & $4.000 \pm 5.659$ & 0.839 & 0.992 \\
\hline $1,000-5,000$ yuan & $2.493 \pm 2.631$ & & $4.296 \pm 2.826$ & & $0.004^{\star \star}$ \\
\hline $5,000-1,0000$ yuan & $4.529 \pm 3.105$ & & $3.964 \pm 2.099$ & & 0.513 \\
\hline Over 1,0000 yuan & $3.167 \pm 3.792$ & & $3.619 \pm 2.439$ & & 0.656 \\
\hline \multicolumn{6}{|c|}{ 5.Current family location } \\
\hline Urban & $2.696 \pm 3.331$ & 0.181 & $3.787 \pm 2.430$ & 0.183 & $0.037^{\star}$ \\
\hline Rural & $3.471 \pm 3.471$ & & $4.706 \pm 2.710$ & & 0.175 \\
\hline
\end{tabular}

${ }^{*} P<0.05, " P<0.01$ by independent samples- $T$ test or one-way ANOVA.

extent. As gender differences have been frequently studied when it came to psychology, it has been demonstrated that females were more likely to experience appearance dissatisfaction (19) and more at risk for emotional distress than males with the same diagnosis due to their higher awareness of sentiment and sensitivity (20).

Moreover, a statistical difference in patients who were an only child was found between CL/P patients and the general. For the sake of reasonable explanation, we found that as parents with children or children with siblings suffering from severe diseases such as cancer were more likely to break the family's normal function and dynamic balance (21), dysfunctional family predicted higher levels of anxiety and other emotional disorders in adolescents, receiving more negative feedback and discouragement of emotional expression (22). However, opposite outcomes have been reported in our study. It's worth noting that even though China's one-child policy ended in 2015, it was in place for over three decades and was mainstream in Chinese families, contributing to the psychosocial differences between groups of only children vs. children with siblings. We found that non-only-children with CL/P scored higher than only-child patients, implying that having siblings might be a risk correlated with anxiety, depression and other psychological disorders (23), partially explained by the so-called resource dilution model (24), meaning that increase in numbers of children bringing about a reduction in available resources from parent to child (25). As for children in general, opposite outcomes have been reported, indicating the complex predictive effects on anxiety in an only child vs. a non-only child group.
In terms of household income, CL/P patients with a monthly household income between 5,000 and 10,000 yuan were documented to have the highest GAD-7 scores, followed by a monthly household income lower than 1,000 yuan. A statistically significant difference in scores was found between groups in subjects with monthly household income between 1,000 and 5,000 yuan, as the control group scored higher than the study group. Given that the general consensus from most published studies was that the severity of anxiety and depression was negatively associated with the household income $(26,27)$, socioeconomic deprivation could be intricately linked to mental illness. Although low family income implied poor access to healthcare, less educational opportunities, increased risk of unemployment and eventually giving rise to mental illnesses, $\mathrm{CL} / \mathrm{P}$ patients tend to exhibit stronger resilience in the face of adversity, with enhanced ability to cope and manage stress and negative experiences compared to the general population due to their craniofacial anomaly (17), which might result in a lower degree of anxiety caused by unsatisfied economic conditions. Anyway, a deeper study should be implemented in the future to investigate the specific relationship between economic conditions and CL/P patients' psychological status.

Higher GAD-7 scores were obtained between groups in subjects from rural areas, which could be partly explained by the negative role of their residential location that could influence their self-worth and self-knowledge (28). It is thought that living in undeveloped areas is an essential factor in deciding one's inner awareness and it can cause more psychological distress (29). In our study, a statistical difference in GAD-7 scores was also found 
between CL/P patients and people without CL/P of the urban area, while the latter scored much higher.

Several limitations need to be noted in our study. The participants of our study were recruited from a limited geographic area and the study was conducted in only one specialized hospital of stomatology in western China. Also, the sample size of our study was not large enough. In addition, there was the outbreak of The 2019 Coronavirus Disease (COVID19) epidemic during our collection of data, posing a challenge to psychological resilience upon both Chinese society and the international community. Our results might be influenced by the outbreak and prevalence of COVID-19.

\section{CONCLUSION}

Herein, given the limitations of this study, we preliminarily provided some evidence on the significance of various causes of anxiety in Chinese CL/P patients. Most importantly, we found that the severity of anxiety in Chinese CL/P patients was not severer than those without CL/P.

\section{DATA AVAILABILITY STATEMENT}

The original contributions presented in the study are included in the article/supplementary material, further inquiries can be directed to the corresponding authors.

\section{REFERENCES}

1. Volpicelli EJ, Pfaff MJ, Potemra HMK, De Leon FS, Lin J, Ayeroff JR, et al. Discrepancies in parent perceptions and patient-reported psychosocial function in children with craniofacial anomalies. Plastic Reconst Surgery. (2020) 145:175-83. doi: 10.1097/prs.0000000000006388

2. Millard T, Richman LC. Different cleft conditions, facial appearance, and speech: relationship to psychological variables. Cleft Palate Craniofac J. (2001) 38:68-75. doi: 10.1597/1545-1569_2001_038_0068_dccfaa_2.0.co_2

3. Costa B, Ardouin K, Stock N. Factors associated with psychological adjustment in adults with cleft lip and/or palate: findings from a national survey in the United Kingdom. Cleft Palate Craniofac J. (2021) 2021:10556656211028494. doi: 10.1177/10556656211028494

4. Shapiro DN, Waljee J, Ranganathan K, Buchman S, Warschausky S. Using the patient reported outcomes measurement information system to evaluate psychosocial functioning among children with craniofacial anomalies. Plastic Reconst Surgery. (2015) 135:1673-9. doi: 10.1097/prs.0000000000001269

5. Craske MG, Rauch SL, Ursano R, Prenoveau J, Pine DS, Zinbarg RE. What is an anxiety disorder? Dep Anxiety. (2009) 26:1066-85. doi: 10.1002/da.20633

6. Nicholls W, Harper C, Robinson S. Data linkage: cleft live-birth prevalence and hospitalizations in Western Australia: 1980 to 2016. Cleft Palate Craniofac J. (2020) 57:1155-65. doi: 10.1177/1055665620943423

7. Sun J, Liang K, Chi X, Chen S. Psychometric properties of the generalized anxiety disorder scale-7 item (GAD-7) in a large sample of Chinese adolescents. Healthcare. (2021) 9:1709. doi: 10.3390/healthcare9121709

8. Spitzer RL, Kroenke K, Williams JB, Löwe B. A brief measure for assessing generalized anxiety disorder: the GAD-7. Arch Int Med. (2006) 166:10927. doi: 10.1001/archinte.166.10.1092

9. He Xiaoyan LC, Qian J, Cui H, Wu W. Reliability and validity of a generalized anxiety scale in general hospital outpatients. Shanghai Arch Psychiatry. (2010) 22:200-3. doi: 10.3969/j.issn.1002-0829.2010.04.002

\section{ETHICS STATEMENT}

The studies involving human participants were reviewed and approved by the Ethics Committee of West China Hospital of Stomatology, Sichuan University (No. WCHSIRB-D-2016084R1). Written informed consent to participate in this study was provided by the participants' legal guardian/next of kin. Written informed consent was obtained from the individual(s), and minor(s)' legal guardian/next of kin, for the publication of any potentially identifiable images or data included in this article.

\section{AUTHOR CONTRIBUTIONS}

ZX and CY contributed equally to this work. ZX, CY, YZ, YY, WX, and YZ contributed to the collection of data. ZX, CY, YZ, and TC analyzed the data. ZX, CY, BS, HH, and CG contributed to writing and revising the paper. $\mathrm{BS}, \mathrm{HH}$, and $\mathrm{CG}$ supervised the research. All authors contributed to the article and approved the submitted version.

\section{FUNDING}

This work was supported by the Research and Develop Program, West China Hospital of Stomatology Sichuan University (RD-02202107), granted to $\mathrm{HH}$.
10. Larsson P, Bondemark L, Häggman-Henrikson B. The impact of oro-facial appearance on oral health-related quality of life: a systematic review. J Oral Rehabil. (2021) 48:271-81. doi: 10.1111/joor.12965

11. Nicholls W, Harper C, Robinson S, Persson M, Selvey L. Adult-specific life outcomes of cleft lip and palate in a Western Australian cohort. Cleft Palate Craniofac J. (2018) 55:1419-29. doi: 10.1177/1055665618768540

12. Lima LS, Ribeiro GS, Aquino SN, Volpe FM, Martelli DR, Swerts MS, et al. Prevalence of depressive symptoms in patients with cleft lip and palate. Brazil J Otorhinolaryngol. (2015) 81:177-83. doi: 10.1016/ j.bjorl.2015.01.004

13. Feragen KB, Stock NM. When there is more than a cleft: psychological adjustment when a cleft is associated with an additional condition. Cleft Palate Craniofac J. (2014) 51:5-14. doi: 10.1597/12-328

14. Feragen KB, Stock NM, Sharratt ND, Kvalem IL. Self-perceptions of romantic appeal in adolescents with a cleft lip and/or palate. Body Image. (2016) 18:143-52. doi: 10.1016/j.bodyim.2016.06.009

15. Hansen-Moore JA, Kapa HM, Litteral JL, Nahata L, Indyk JA, Jayanthi VR, et al. Psychosocial functioning among children with and without differences of sex development. J Pediatric Psychol. (2021) 46:69-79. doi: 10.1093/jpepsy/jsaa089

16. de Oliveira RL, de Santana Santos T, de Almeida Teixeira JL, Martins-Filho PR, da Silva LC. Health-related quality of life patients with a cleft lip and/or palate. J Craniofac Surgery. (2015) 26:2315-9. doi: 10.1097/scs.0000000000001992

17. Feragen KB, Borge AI, Rumsey N. Social experience in 10-year-old children born with a cleft: exploring psychosocial resilience. Cleft Palate Craniofac J. (2009) 46:65-74. doi: 10.1597/07-124.1

18. Boyce JO, Kilpatrick N, Morgan AT. Speech and language characteristics in individuals with nonsyndromic submucous cleft palate-A systematic review. Child Care Health Dev. (2018) 44:818-31. doi: 10.1111/ cch. 12613 
19. Turk F, Kellett S, Waller G. Determining the potential link of selfcompassion with eating pathology and body image among women: a longitudinal mediational study. Eating Weight Dis. (2021) 26:2683-91. doi: 10.1007/s40519-021-01144-1

20. Paxton SJ, Neumark-Sztainer D, Hannan PJ, Eisenberg ME. Body dissatisfaction prospectively predicts depressive mood and low selfesteem in adolescent girls and boys. J Clin Child Adol Psychol. (2006) 35:539-49. doi: 10.1207/s15374424jccp3504_5

21. Van Schoors M, De Paepe AL, Lemiere J, Morez A, Norga K, Lambrecht K, et al. Family adjustment when facing pediatric cancer: the role of parental psychological flexibility, dyadic coping, and network support. Front Psychol. (2019) 10:2740. doi: 10.3389/fpsyg.2019.02740

22. Chi X, Huang L, Wang J, Zhang P. The prevalence and socio-demographic correlates of depressive symptoms in early adolescents in china: differences in only child and non-only child groups. Int J Environ Res Public Health. (2020) 17:10-1. doi: 10.3390/ijerph17020438

23. Cameron L, Erkal N, Gangadharan L, Meng X. Little emperors: behavioral impacts of China's one-child policy. Science. (2013) 339:953-7. doi: 10.1126/science. 1230221

24. Kalmijn $M$, van de Werfhorst $H$. Sibship size and gendered resource dilution in different societal contexts. PLoS ONE. (2016) 11:e0160953. doi: 10.1371/ journal.pone.0160953

25. Cao Y, Huang L, Si T, Wang NQ, Qu M, Zhang XY. The role of onlychild status in the psychological impact of COVID-19 on mental health of Chinese adolescents. J Affect Dis. (2021) 282:316-21. doi: 10.1016/ j.jad.2020.12.113

26. Huang M, Liu Y, Wang J, Mo L, Wang Y, Chen L, et al. High rates of depression anxiety and suicidal ideation among inpatients in general hospital in China. Int J Psychiatry Clin Practice. (2019) 23:99105. doi: 10.1080/13651501.2018.1539179
27. Finegan M, Firth N, Delgadillo JJ, PrjotSfPR. Adverse impact of neighbourhood socioeconomic deprivation on psychological treatment outcomes: the role of area-level income and crime. Psychother Res. (2020) 30:546-54. doi: 10.1080/10503307.2019.1649500

28. Wei L, Ge C, Xiao W, Zhang X, Xu J. Cross-sectional investigation and analysis of anxiety and depression in preoperative patients in the outpatient department of aesthetic plastic surgery in a general hospital in China. JPRAS. (2018) 71:1539-46. doi: 10.1016/j.bjps.2018.07.015

29. Valikhani A, Goodarzi MA. Contingencies of self-worth and psychological distress in iranian patients seeking cosmetic surgery: integrative self-knowledge as mediator. Aesthetic Plastic Surgery. (2017) 41:955-63. doi: 10.1007/s00266-017-0853-8

Conflict of Interest: The authors declare that the research was conducted in the absence of any commercial or financial relationships that could be construed as a potential conflict of interest.

Publisher's Note: All claims expressed in this article are solely those of the authors and do not necessarily represent those of their affiliated organizations, or those of the publisher, the editors and the reviewers. Any product that may be evaluated in this article, or claim that may be made by its manufacturer, is not guaranteed or endorsed by the publisher.

Copyright (c) 2022 Xie, Yang, Zhao, Yang, Xia, Zong, Chi, Shi, Huang and Gong. This is an open-access article distributed under the terms of the Creative Commons Attribution License (CC BY). The use, distribution or reproduction in other forums is permitted, provided the original author(s) and the copyright owner(s) are credited and that the original publication in this journal is cited, in accordance with accepted academic practice. No use, distribution or reproduction is permitted which does not comply with these terms. 